\title{
Correction to: Reference ranges of left ventricular structure and function assessed by contrast-enhanced cardiac MR and changes related to ageing and hypertension in a population-based study
}

\author{
Robin Bülow ${ }^{1}$ (D) Till Ittermann ${ }^{2} \cdot$ Marcus Dörr $^{3,4} \cdot$ Axel Poesch $^{3} \cdot$ Sönke Langner ${ }^{1} \cdot$ Henry Völzke $^{2,4}$. \\ Norbert Hosten ${ }^{1} \cdot$ Marc Dewey $^{5}$
}

Published online: 19 April 2018

(C) European Society of Radiology 2018

\section{Correction to: Eur Radiol \\ https://doi.org/10.1007/s00330-018-5345-y}

The original version of this article, published on 14 March 2018, unfortunately contained a mistake. The following correction has therefore been made in the original:

The sequence of the author names was incorrect, Robin Bülow was mentioned twice. The corrected author list is given above. The original article has been corrected.

The online version of the original article can be found at https://doi.org/ 10.1007/s00330-018-5345-y

\footnotetext{
Robin Bülow

buelowr@uni-greifswald.de

1 Institute for Diagnostic Radiology and Neuroradiology, University Medicine, Ernst Moritz Arndt University Greifswald, Ferdinand-Sauerbruch-Straße 1, 17475 Greifswald, Germany

2 Institute for Community Medicine, SHIP/Clinical-Epidemiological Research, University Medicine, Ernst Moritz Arndt University Greifswald, Walther Rathenau Str. 48, 17475 Greifswald, Germany

3 Department of Internal Medicine B, Cardiology, Pneumology, Infectious Diseases, Intensive Care Medicine, University Medicine, Ernst Moritz Arndt University, Ferdinand-Sauerbruch-Straße 1, 17475 Greifswald, Germany

4 DZHK (German Centre for Cardiovascular Research), Greifswald, Germany

5 Institute for Radiology, Charité Medical School, Charitéplatz 1, 10117 Berlin, Germany
} 